
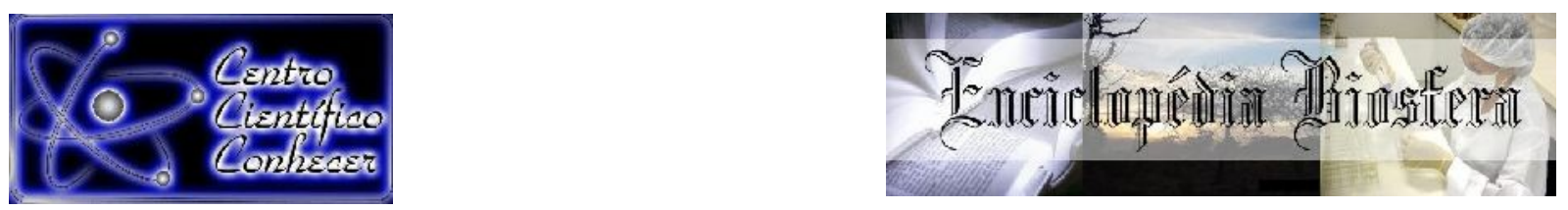

\title{
ANÁLISE INVESTIGATIVA UTILIZADA NO SETOR RODOVIÁRIO PARA CRIAÇÃO DE UM BANCO DE DADOS: SEGMENTO DA BR-135/MA
}

\footnotetext{
Jorcelan Pereira da Rocha ${ }^{1}$, Fernando Vasconcelos Borba ${ }^{2}$

1 Engenheiro civil. Pós-graduando em Infraestrutura de Transportes e Rodovias pelo Instituto de graduação e pós-graduação - IPOG (jorcelan20@gmail.com) Barreirinhas, Maranhão, Brasil.

2 Engenheiro civil. Mestrando em Estruturas e Construção Civil pela Universidade Federal do Pará UFPA - Belém, Pará, Brasil.

Recebido em: 04/10/2019 - Aprovado em: 30/11/2019 - Publicado em: 15/12/2019 DOI: 10.18677/EnciBio_2019B1
}

\begin{abstract}
RESUMO
Este trabalho foi realizado com a finalidade de mostrar que a coleta de informações é indispensável no setor rodoviário, pois consiste em uma busca contínua que possibilita a caracterização de rodovias, reunindo em um banco de dados, entre outras, o histórico da rodovia, condição do pavimento e o estado do sistema de drenagem. Para isso realizou-se o estudo em um trecho de 25,6 km na BR-135/MA, através da análise dos métodos investigativos usados como critério de avaliação de pavimentos rodoviários. A coleta de dados para o estudo ocorreu por meio do auxílio do Departamento Nacional de Infraestrutura de Transportes (DNIT). Esses elementos irão ser atualizados e servirão em futuras estratégias de Manutenção e Restauração (M\&R). Sendo assim, este trabalho permitiu comprovar que é possível a formação de um banco de dados preciso no decorrer dos anos, e apesar de não ter por objetivo avaliar o trecho em estudo, destaca de forma simples e precisa, através das bibliografias, não só os subsídios, mas também, os equipamentos e métodos utilizados em campo para tal investigação.
\end{abstract}

PALAVRAS-CHAVE: Banco de dados. Informações. Setor rodoviário.

\section{INVESTIGATIVE ANALYSIS USED IN THE ROAD SECTOR FOR CREATING A DATABASE: SEGMENT OF BR-135 / MA}

\section{ABSTRACT}

This work was carried out with the purpose of showing that the collection of information is indispensable in the road sector, as it consists of a continuous search that enables the characterization of highways, gathering in a database, among others, the history of the highway, condition of the floor and the state of the drainage system. For this, the study was carried out in a $25.6 \mathrm{~km}$ stretch on BR- 
135 / MA, through the analysis of the investigative methods used as a criterion for road pavement evaluation. Data collection for the study took place through the help of the National Department of Transport Infrastructure (DNIT). These elements will be updated and will serve in future Maintenance and Restoration (M\&R) strategies. Thus, this work showed that it is possible to form an accurate database over the years, and despite not having the objective of evaluating the section under study, it highlights simply and precisely, through bibliographies, not only the subsidies, but also the equipment and methods used in the field for such research.

KEYWORDS: Information. Road sector. Database.

\section{INTRODUÇÃO}

No Brasil, dos 57,2 mil quilômetros de rodovias federais pavimentadas que são de responsabilidade do Departamento Nacional de Infraestrutura de Transportes (DNIT), 33,7 mil (59\%) encontram-se em boa condição de conservação. Ainda $18 \%$ das rodovias encontram-se em condição regular; $10 \%$, ruim; e 13\%, péssimo (DNIT, 2018).

É sabido que a interferência do transporte rodoviário na economia brasileira é muito elevada, pois o modal rodoviário é responsável pela maioria das movimentações, sejam de carga $(61,1 \%)$ ou passageiros $(95 \%)$, tornando-se indispensável em aspectos socioeconômicos (CNT, 2016).

Para ODA (2016), nas atividades de Manutenção e Restauração (M\&R) os métodos avaliativos, como: Método da Matriz, Árvore de Decisão, Método do Custo do Ciclo de Vida e Método de Otimização, são fundamentais para uma futura tomada de decisão, entretanto, depende da quantidade de elementos coletados no decorrer da vida útil de uma rodovia.

A maioria dos administradores rodoviários concluem que a coleta de dados é um componente muito importante para os valores totais relacionados com a conservação de pavimentos asfálticos no passar do tempo. Tal coleta é iniciada depois das fases de planejamento e treinamento dos técnicos que fazem as visitas em campo. Assim, para maior eficiência dos serviços, existe uma coordenação de pesquisa que tem por objetivo acompanhar a coleta e receber os dados parciais; e validar as informações coletadas. Ressalta-se que os pesquisadores não impõem notas ou referências aos trechos analisados, eles só identificam os atributos de campo no formulário apropriado (CNT, 2018). Assim, procura-se desenvolver e utilizar uma maior quantidade de tecnologias que possibilitam que essa coleta em campo seja feita com a maior rapidez possível e com mínima influência no tráfego da rodovia. Percebe-se que esse processo é muito importante, porquanto através da ocorrência é possível a aquisição de fontes de embasamento para tomada de decisão. Lembrando que as informações devem ser obtidas com objetividade, atualidade e confiabilidade (DNIT, 2011).

Para o DNIT (2005) as etapas de coleta de dados devem ser: Descrição do projeto e apresentação à Diretoria Geral e à Diretoria Colegiada; Termo de referência; Edital/Licitação; Contratação; Levantamentos de campo; Tratamento em escritório; Disponibilização das informações processadas. Após a obtenção dos dados já coletados, de acordo com a CNT (2018), é realizada a análise da 
consistência, possibilitando a obtenção dos resultados necessários para a consolidação do armazenamento para todos os trechos em pesquisa, ou seja, o objetivo é reunir os subsídios em um único local, para uma futura observação de correção, que se fundamenta na verificação da extensão total pesquisada.

Outro fator importante, de acordo com Zofka (2018), é a análise do pavimento em questões climáticas não favoráveis, pois as muitas condições do pavimento são atingidas de distintas maneiras e estão relacionadas diretamente com o desempenho do pavimento. Logo, é preciso utilizar modelos que capturam ao máximo o real comportamento referente a fatores climáticos, como temperatura e precipitação.

Dessa maneira, existe um entendimento geral, em meio aos pesquisadores de campo, que mostra a dificuldade em assimilar, no período de graduação em engenharia, as principais formas de coleta de elementos com objetivo de uma avaliação rodoviária, assim, este trabalho significa de forma resumida uma análise positiva quanto às informações coletadas sobre o histórico da rodovia, avaliação do pavimento e estado do sistema de drenagem.

Esta pesquisa consistiu em destacar não só os subsídios coletados e armazenados, mas também teve por objetivo demonstrar alguns dos principais métodos e equipamentos encontrados em referências bibliográficas disponíveis no ambiente acadêmico para área de transportes. Sendo assim, não se buscou aferir o trecho em estudo, mas mostrar as informações obtidas e as diferentes formas de obtenção para um segmento rodoviário de $25,6 \mathrm{~km}$ na BR-135 no estado do maranhão.

\section{MATERIAL E MÉTODOS}

A pesquisa foi concluída no ano de 2018, no Estado do Maranhão, onde se realizou uma coleta de dados sobre um trecho de 25,6 km da Rodovia BR-135. Inicialmente, procedeu-se com pesquisa nos documentos fornecidos pela Superintendência Regional do DNIT no estado do Maranhão com a finalidade de coletar todas as informações históricas do segmento analisado. Posteriormente, efetuaram-se investigações in loco na rodovia com a finalidade de coletar as principais características.

\section{Área de estudo}

Possuindo uma extensão de 2.432,00 quilômetros, a BR-135 é de extrema importância para o território nacional, pois é a única rodovia que liga Belo Horizonte (MG) com São Luís (MA). Entretanto, o trecho em estudo é formado por $25,6 \mathrm{Km}$ (Santa Rita / Povoado de Outeiro), nas seguintes coordenadas geográficas: (3॰8'42.10" S / 44¹9’29.33" O) e (3॰19’53.80" S / 44²6’49.69" O).

Na Figura 1, observa-se a localização do trecho em análise. 


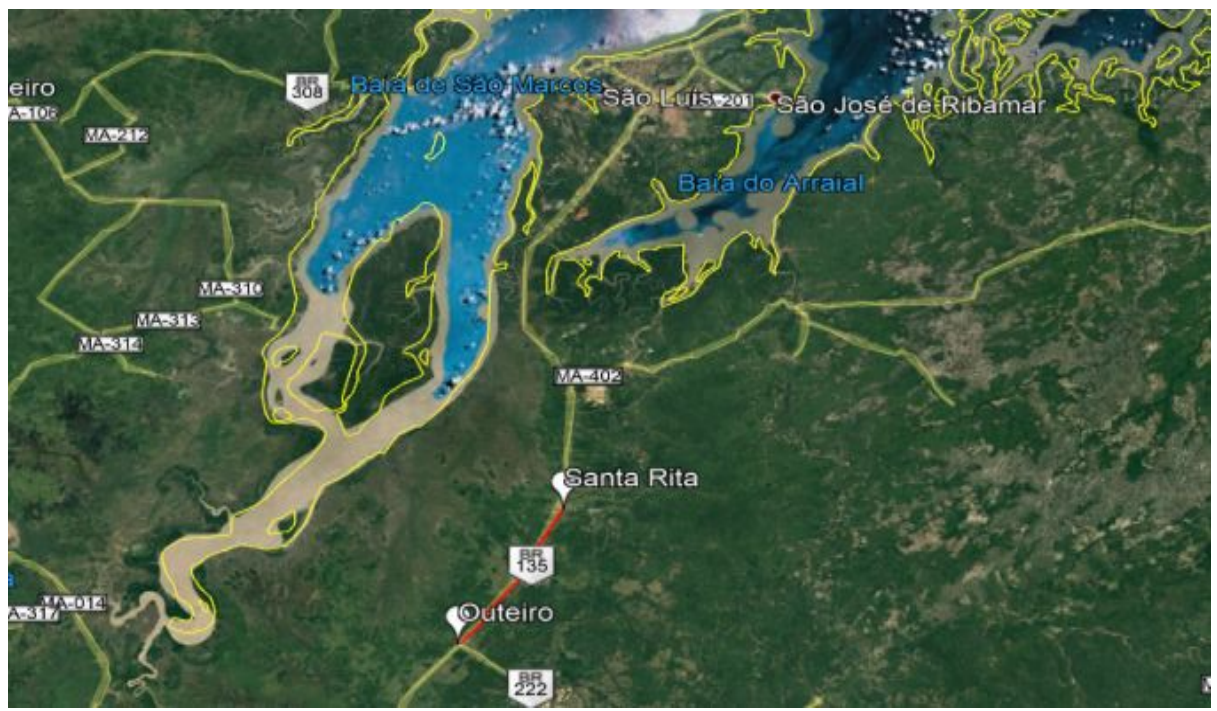

FIGURA 1 - Trecho da BR-135 em estudo

Fonte: Adaptada de Google Earth (2018)

\section{EQUIPAMENTOS E MÉTODOS UTILIZADOS NA PESQUISA}

No primeiro passo do estudo foram coletadas informações sobre o histórico da rodovia, como: características regionais da rodovia; datas de implantação; datas de pavimentação; datas de melhoramentos; datas dos serviços de manutenção; e demais informações encontradas nos projetos de engenharia utilizadas para concepção da via.

Já no segundo passo, foram realizadas visitas no trecho durante o decorrer dos anos de 2017 e 2018. Assim, para obtenção dos resultados foram concretizados alguns levantamentos in loco com a finalidade de completar o banco de dados. De maneira geral, todos os métodos e equipamentos utilizados na obtenção dos elementos que compõe o grupo de informações coletadas são descritos da seguinte forma:

a. $\mathrm{Na}$ avaliação realizada com a finalidade de diagnosticar as patologias na camada de rolamento foram obtidas: área de trincamento, área de desgaste, número de buracos, área de bordo quebrada, trilha de roda média e profundidade da textura.

b. Para coleta de informações referentes ao estado do sistema de drenagem, foram feitas inspeções no local que quantificaram a degradação de todos os elementos que o compõe. Com as informações obtidas, tornou-se possível classificar tal sistema como "ruim" utilizando critérios do DNIT.

c. Para obtenção do Índice de Irregularidade Internacional (IRI) e o Levantamento Visual Contínuo (LVC) foi utilizado o Veículo de Diagnóstico Rodoviário que, de acordo com o DNIT, (2014), devido a grande necessidade na busca por elementos de forma mais rápida e precisa, esse veículo foi criado. Assim, a partir do momento da implantação desse veículo, foi possível alcançar uma mais perfeita relação custo e benefício, além de produzir dados com propriedades mais precisas. 
Observa-se na Figura 2, o veículo de diagnóstico foi idealizado desde 2001 e posto em integral funcionamento em 2012.

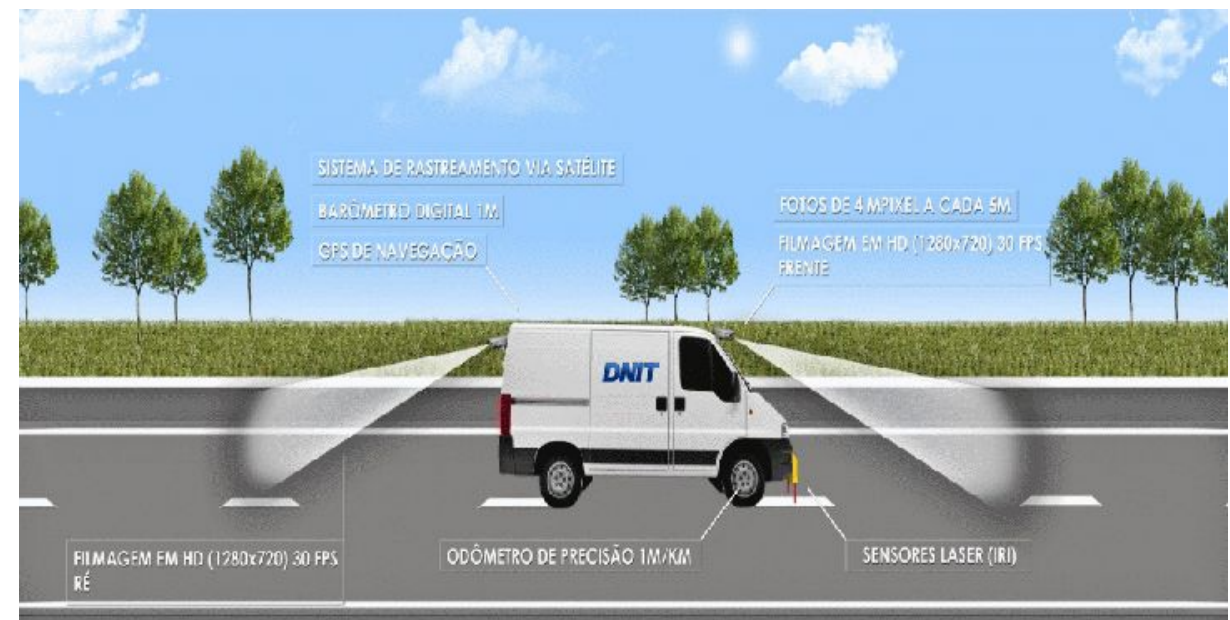

FIGURA 2 - Veículo Diagnóstico

Fonte: DNIT (2014)

d. Na coleta de dados sobre a condição operacional do pavimento foi realizada uma pesquisa nos documentos disponíveis do DNIT, foi obtido Volume Médio Diário Anual de Tráfego (VMD), que conforme o DNIT (2006) é feita em termos de capacidade de tráfego, sendo uma avaliação volumétrica que tem como referência dados já catalogados para determinado trecho. Uma vez que, é fundamental à utilização de informações obtidas por meio de contagens realizadas em anos passados, objetivando, a verificação de um suposto crescimento de tráfego no decorrer do tempo.

e. Na coleta de dados sobre a condição estrutural do pavimento foi aplicado o uso da Viga Benkelman, (Figura 3), segundo Balbo (2007), é um equipamento que tem por objetivo medir deflexões nos pavimentos e através dessas determinações é possível traçar uma linha de influência longitudinal da carga, também denominada bacia de deflexões.

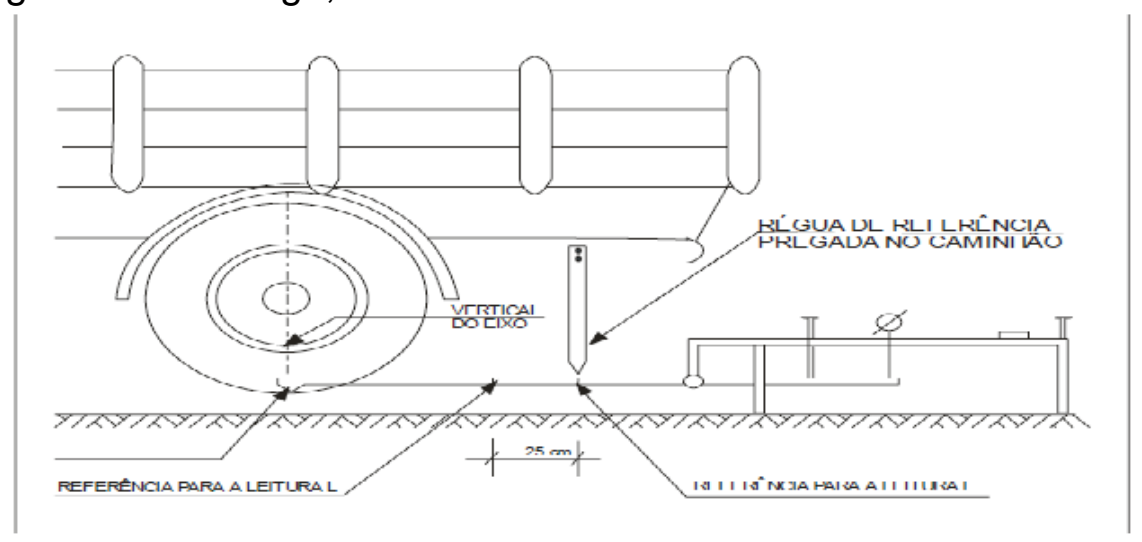

FIGURA 3 - Posicionamento da viga Benkelman e da prova de carga Fonte: DNIT (2006) 


\section{RESULTADOS E DISCUSSÃO}

Apesar da análise ser em um pequeno trecho da BR-135/MA, chama-se atenção para o fato de que durante a busca por subsídios, houve grande dificuldade na coleta de elementos que representassem a real situação da rodovia. Entretanto, através das informações obtidas in loco/escritório foi possível destacar as necessidades do trecho, o que permitiu a gerência das intervenções necessárias no decorrer dos anos. Assim, através das pesquisas, foram muitas as informações obtidas sobre o determinado trecho rodoviário, que após serem agrupadas, serviram para criar parâmetros que segundo a CNT (2018) favorecem a classificação das rodovias, de acordo com a avaliação executada por unidades de pesquisa, que auferem notas relacionadas às condições de cada uma das características observadas em campo.

Chama-se a atenção para relevância das informações coletadas e fornecidos pelo DNIT para os $25,6 \mathrm{Km}$ analisados, entretanto, como é manifestado na Figura 4, às visitas realizadas em campo foram os passos mais importantes da pesquisa, pois possibilitaram a confiabilidade das informações que compõe o banco de dados formado com o estudo.

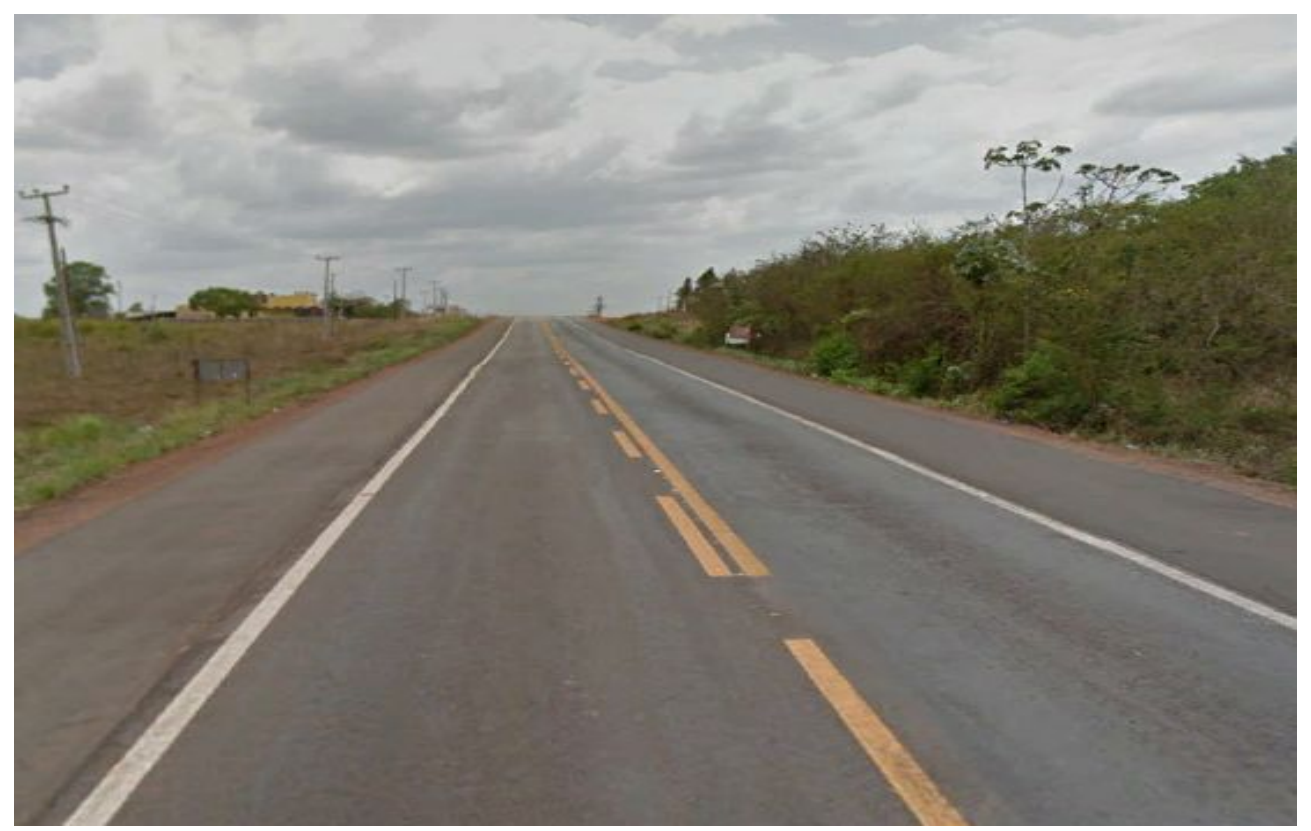

FIGURA 4 - Pavimento asfáltico do trecho em estudo

Fonte: Elaborada pelos autores (2018)

Destaca-se que o armazenamento considerou informações desde 2010, e apesar de alguns elementos precisarem ser atualizadas, a finalidade do estudo é cumprida ao se conseguir a conexão dos dados em apenas um local verificável. Sendo assim, tornou-se possível efetivar a pesquisa através do armazenamento dos elementos que caracterizam o segmento, esses são: Definição e característica do segmento; Geometria do segmento; Dados do pavimento em estudo e Condição do pavimento.

Assim, para concluir a formação do BANCO DE DADOS foram obtidas as seguintes informações: 
a. Definição e característica do segmento;

- Rodovia: 135/MA;

- Segmento: Km 70 ao Km 95,6.

- Extensão: 25,6 km;

- Número de identificação da seção (PNV): 135BMA0080;

- Tipo de fluxo: Rodovia padrão de duas faixas;

- Tipo de fluxo de tráfego: Fluxo Livre;

- Tipo de clima: Tropical Úmido;

- Classe da rodovia: Primária;

- Tipo de superfície: Pavimento betuminoso;

- Tipo de pavimento: Concreto asfáltico sobre base granular;

- Número de faixas: 2;

- VMD: 17683;

- Ano da última selagem, recapeamento ou restauração: 2016;

- Sentido do tráfego: Dois sentidos.

b. Geometria do segmento;

- Largura da pista: 7,2 m;

- Largura do acostamento: 2,0 m;

- Subidas e descidas: 1,00 m/km;

- Grau de curvatura: 3,00 deg/km;

- Velocidade limite: $110 \mathrm{~km} / \mathrm{h}$;

- Altitude: 43 m.

c. Dados do pavimento em estudo;

- CBR médio do subleito do segmento: 4\%;

- Espessura de superfície mais recente: 25 mm;

- Espessura de superfície anterior: 100 mm;

- Ano da última construção/reconstrução: 2010;

- Ano da última reabilitação: 2015;

- Ano da última resselagem: 2015;

- Ano do último tratamento preventivo: 2015.

A Figura 5 expõe a condição do acostamento do segmento analisado.

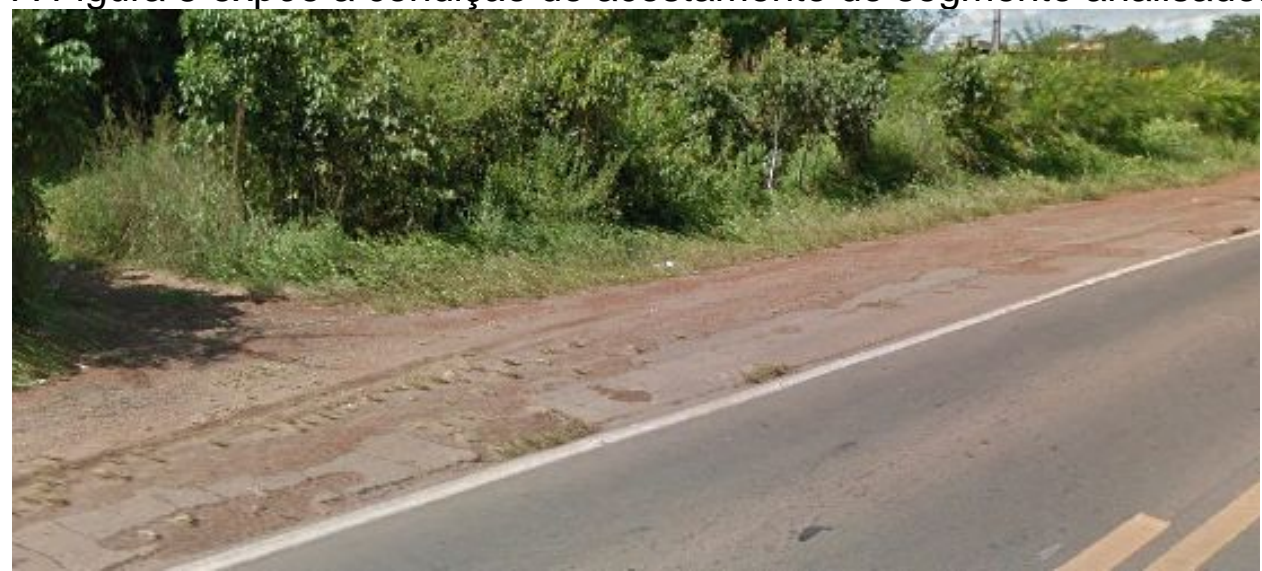

FIGURA 5 - Patologias no acostamento

Fonte: Elaborada pelo autor (2018) 
d. Condição do pavimento.

- Irregularidade (IRI): 6,0 m/km;

- Área de trincamento : $15 \%$;

- Área de desgaste: $20 \%$;

- Número de buracos: $5 / \mathrm{km}$;

- Área de bordo quebrada: $100 \mathrm{~m}^{2} / \mathrm{km}$;

- Trilha de roda média: $15 \mathrm{~mm}$;

- Profundidade da textura: $0,30 \mathrm{~mm}$;

- Resistência à derrapagem $(50 \mathrm{~km} / \mathrm{h}): 0,35$;

- Drenagem: Ruim.

Algumas das patologias localizadas na superfície do pavimento podem ser visualizadas na Figura 6 .

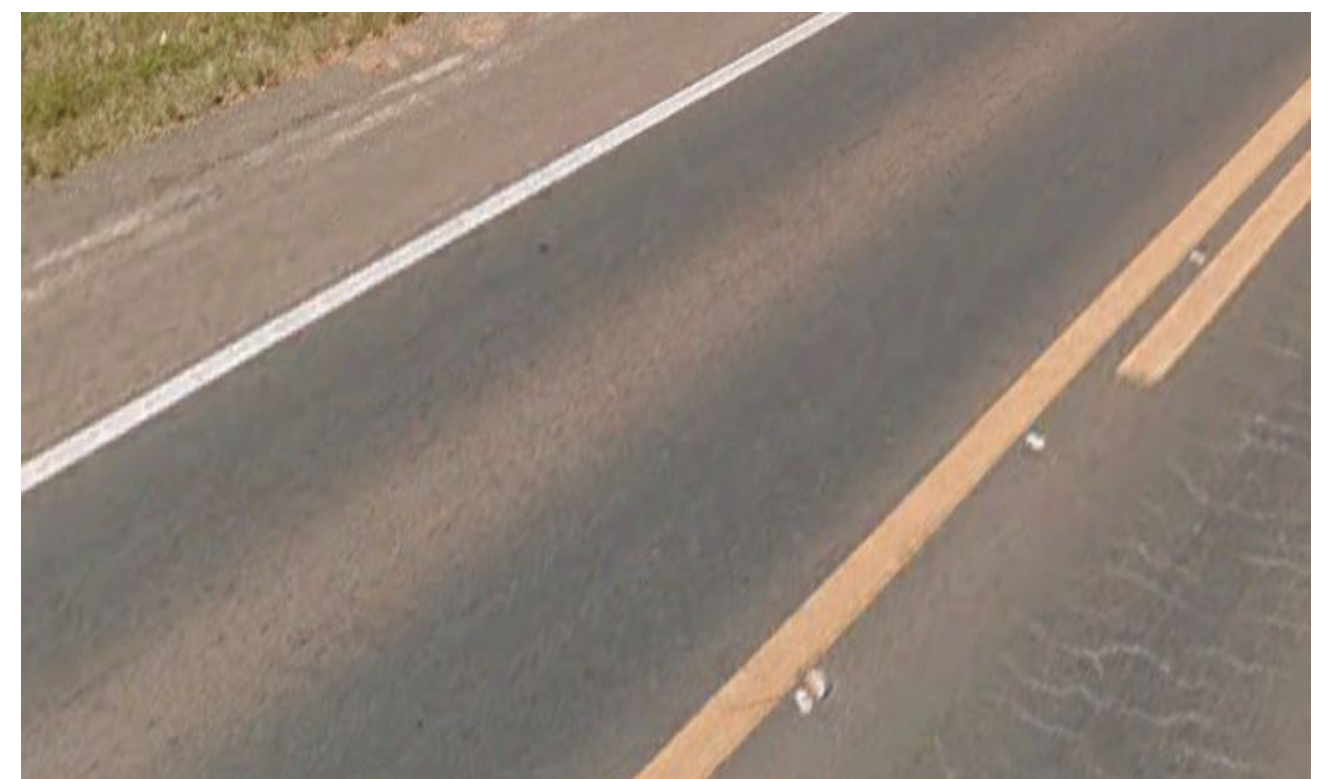

FIGURA 6 - Patologias no pavimento

Fonte: Elaborada pelo autor (2018)

Após o agrupamento das informações, de acordo com o CONSÓRCIO DYNATEST ENGEMAP (2015) no intercâmbio com o banco de dados para formação de um futuro Sistema de Gerência de Pavimentos (SGP) existe um conjunto de componentes que devem interagir de forma unificada e contínua. Sendo um grupo formado por fatores internos como o planejamento, projeto, construção e a manutenção dos pavimentos, e por fatores externos como os recursos orçamentários, elementos necessários ao sistema e diretrizes políticas e administrativas. Assim, torna-se possível a universalização da informação, admitindo que cada especialista faça consultas e análises sugestivas a um trecho ou à malha.

Dessa forma, apesar da formação do banco de dados ser o objetivo principal realizado neste estudo, para uma pesquisa futura, é possível um relatório 
destas análises com os resultados em diferentes categorias, pois as informações coletadas permitem diversas apreciações na condição estrutural, funcional e operacional do pavimento.

\section{CONCLUSÃO}

Perante os resultados obtidos, a formação do banco de dados para possíveis tomadas de decisões mostrou-se como uma solução viável perante a necessidade do DNIT/MA. Porquanto, determinações poderão ser adotadas de forma que respeitem a relação de custo e benefício, uma vez que em todo território nacional existe um desperdício muito grande de recursos aplicados em soluções ineficazes por falta de informações que representem a real situação da rodovia. Com isso, conclui-se que esta pesquisa deixa um grupo de elementos armazenados e acessíveis, ou seja, é possível, com o emprego de programas para processamento, a análise dos dados e a emissão de relatórios e/ou gráficos, como extração do sistema, amparando os envolvidos nas atividades de Manutenção e Restauração (M\&R) adotadas para segmento estudado.

\section{REFERÊNCIAS}

BALBO, J.T. Pavimentação asfáltica: materiais, projeto e restauração. São Paulo: Oficina de Textos, 2007.

CONSÓRCIO DYNATEST ENGEMAP. Relatório técnico: catálogo de soluções de manutenção para pavimentos flexíveis. 2015.

CNT - CONFEDERAÇÃO NACIONAL DE TRANSPORTE. Metodologia. Confederação Nacional de Transportes. 2018. Disponível em: $<$ https://pesquisarodovias.cnt.org.br/Paginas/metodologia>. Acesso em 08/05/2019

CNT - CONFEDERAÇÃO NACIONAL DE TRANSPORTES. Pesquisa CNT de Rodovias 2016 - Relatório Gerencial. Confederação Nacional de Transportes. 2016. Disponível em: <http://pesquisarodovias.cnt.org.br>. Acesso em 10/12/2018.

\section{DNIT - DEPARTAMENTO NACIONAL DE INFRAESTRUTURA DE TRANSPORTES. ICM 2018. 2018. Disponível em: <http://www.dnit.gov.br/noticias/icm-2018-indice-que-avalia-as-rodovias-federais- pavimentadas-e-divulgado>. Acesso em 20/05/2019}

DNIT - DEPARTAMENTO NACIONAL DE INFRAESTRUTURA DE TRANSPORTES. Manual de conservação rodoviária. Rio de Janeiro, 2005.

DNIT - DEPARTAMENTO NACIONAL DE INFRAESTRUTURA DE TRANSPORTES. Manual de gerência de pavimentos. Rio de Janeiro, 2011.

DNIT - DEPARTAMENTO NACIONAL DE INFRAESTRUTURA DE TRANSPORTES. Manual de restauração de pavimentos asfálticos. Rio de 
Janeiro, 2006.

DNIT - DEPARTAMENTO NACIONAL DE INFRAESTRUTURA DE TRANSPORTES. Veículo de Diagnóstico de Rodovias: Maior eficiência e economia para o Sistema de Gerenciamento de Pavimento da malha rodoviária federal. 2014.

ODA, S. Notas de Aula. Disciplina EER 555 Pavimentação A. Departamento de Engenharia de Transportes. Escola Politécnica da Universidade Federal do Rio de Janeiro - UFRJ, 2016.

ZOFKA, A. Proactive pavement asset management with climate change aspects. Warszawa, 2018.2 Disponível em: $<$ https://iopscience.iop.org/article/10.1088/1757-899X/356/1/012005>. doi:10.1088/1757-899X/356/1/012005. Acesso em 07/02/2019. 